\title{
Two-photon and two-gluon decays of $p$-wave heavy quarkonium using a covariant light-front approach
}

\author{
Chien-Wen Hwang* and Rurng-Sheng Guo ${ }^{\dagger}$ \\ Department of Physics, National Kaohsiung Normal University, \\ Kaohsiung, Taiwan 824, Republic of China
}

\begin{abstract}
In this paper, a study of two-photon and two-gluon decays in the context of $p$-wave heavy quarkonia is presented. Within the covariant light-front framework, the annihilation rates of scalar and tensor quarkonium states are derived. In the absence of free parameters in this case, the results for the charmonium decay widths are consistent with the experimental data. However, in comparison to other theoretical calculations, there are large discrepancies in our results regarding bottomonia.
\end{abstract}

PACS numbers: 14.40.Pq, 12.39.Ki

\footnotetext{
*t2732@nknucc.nknu.edu.tw

† t1953@nknucc.nknu.edu.tw
} 


\section{INTRODUCTION}

Heavy quarkonium provides for a unique laboratory to study quantum chromodynamics (QCD) regarding the bound states of a heavy quark-antiquark system. Notably, the twophoton and two-gluon annihilation rates of $p$-wave heavy quarkonium are helpful for better understanding the details of quark-antiquark interaction and can function as stringent tests for a potential model. Regarding experimentation, the two-photon decay width of $\chi_{c J}$ has been measured by many laboratories [1, 2] and a new CLEO measurement was reported recently [2]. Regarding theory, relevant decay rates were first obtained through nonrelativistic approximation [3, 4]; the relativistic corrections were included within the Bethe-Salpeter equation [5 8], potential model [9], relativistic quark model [10-12], nonrelativistic QCD factorization framework [13], two-body Dirac equations of constraint dynamics [14], effective Lagrangian [15], and bound state perturbation theory [16]. The lattice calculation [17] and rigorous QCD predictions [18, 19] were also applied. In addition, the $s$-wave and $p$ wave electromagnetic and light hadronic quarkonium decays in the heavy-quark velocity expansion were computed in the nonrelativistic QCD approach [20-22].

This paper is aimed at the study of the two-photon and two-gluon decay widths of $p$ wave heavy quarkonium states including the scalar $\left(\chi_{c 0}, \chi_{b 0}, \chi_{b 0}^{\prime}\right)$ and tensor $\left(\chi_{c 2}, \chi_{b 2}, \chi_{b 2}^{\prime}\right)$ mesons. It is known that heavy quarkonium is relevant to nonrelativistic treatments [3], 4, 23]. Although nonrelativistic QCD is a powerful theoretical tool used to separate highenergy modes from low-energy contributions, in most cases those attempting the calculation of low-energy hadronic matrix elements have relied on model-dependent nonperturbative methods. The light-front quark model (LFQM) [24, 25] is a relativistic quark model in which a consistent and fully relativistic treatment of quark spins and the center-of-mass motion can be carried out. This model has many advantages. For example, the light-front wave function is manifestly Lorentz invariant as it is expressed in terms of the momentum fraction variables in analog to the parton distributions in the infinite momentum frame. Moreover, hadron spin can also be correctly constructed using the so-called Melosh rotation [26]. This model is very well suited for studying hadronic form factors. Specifically, as the recoil momentum increases (corresponding to a decreasing $q^{2}$ ), we have to start seriously considering relativistic effects. In particular, at the maximum recoil point $q^{2}=0$ where the final-state particle could be highly relativistic, there is no reason to expect that the 
nonrelativistic quark model is still applicable.

The LFQM has been employed to obtain some physical quantities [27-30]. However, one often calculates a particular component (the plus component) of the associated current matrix element in the LFQM formulation. Because of the lack of relativistic covariance, the results may show some inconsistencies. The usual strategy of taking only the plus component of the current matrix elements will ignore the zero-mode contributions and render the matrix element noncovariant. As a consequence, it is desirable to construct a covariant light-front model that can provide a systematic way of exploring zero-mode effects. Such a covariant model has been constructed in [31] for heavy mesons within the framework of heavy-quark effective theory. Without appealing to the heavy-quark limit, a covariant approach of the light-front model has been put forward for the usual $s$-wave mesons [32], extended to the $p$-wave mesons [33], and employed in the context of the $s$-wave heavy quarkonium [34]. In

this study, the $p$-wave heavy quarkonium is explored through this covariant model. The details and formalism are displayed in the next section.

The remainder of this paper is organized as follows. In Sec. II, the formalism of a covariant light-front model is shown in cases of scalar and tensor quarkonia and the annihilation rates of these $p$-wave heavy quarkonium are derived. In Sec. III, after fixing the parameters which appear in the trial wave function, the numerical results and discussions are presented. Finally, conclusions are given in Sec. IV.

\section{FORMALISM OF A COVARIANT LIGHT-FRONT MODEL}

\section{A. Formalism}

In the conventional light-front framework, the constituent quarks of the meson are required to be on their mass shells (see Appendix A of Ref. [33] for an introduction). Jaus [32] proposed a covariant light-front approach that permits a systematic way of dealing with zero-mode contributions. Physical quantities can be calculated in terms of Feynman momentum loop integrals which are manifestly covariant; this means that the constituent quarks of the bound state are off shell. In principle, this covariant approach will be useful if the vertex functions can be determined by solving the QCD bound state equation. In practice, we have to be content with phenomenological vertex functions, such as those employed in 
the conventional light-front model. Therefore, by using the light-front decomposition of the Feynman loop momentum, say $p_{\mu}$, and integrating out the minus component of the loop momentum $p^{-}$, one moves from the covariant calculation to the light-front one. Moreover, the spectator quark is forced to be on its mass shell after $p^{-}$integration. Consequently, the covariant vertex functions can be replaced by the phenomenological light-front ones.

As stated in passing, in going from the manifestly covariant Feynman integral to the light-front integral, the latter is no longer covariant as it can receive additional spurious contributions proportional to the lightlike four vector $\omega^{\mu}=\left(\omega^{-}, \omega^{+}, \omega_{\perp}\right)=(2,0,0 \perp)$. The undesired spurious contributions can be eliminated by the inclusion of the zero-mode contribution which amounts to performing the $p^{-}$integration. The advantage of this covariant light-front framework is that it allows a systematic way of handling the zero-mode contributions and permits the user to obtain covariant matrix elements.

To begin with, we consider the decay amplitudes given by one-loop diagrams, as shown in Fig. 1 for the two-photon decay of $p$ - wave quarkonium states. The incoming meson has

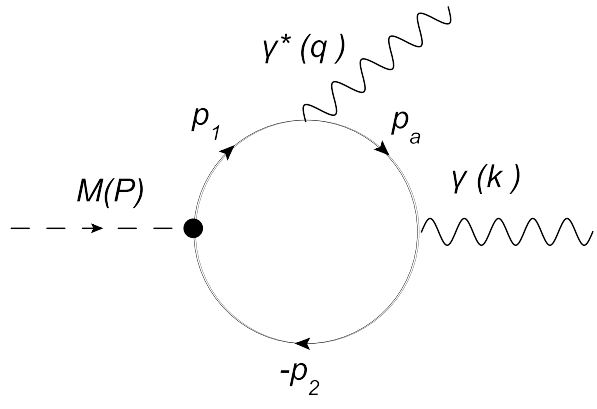

(a)

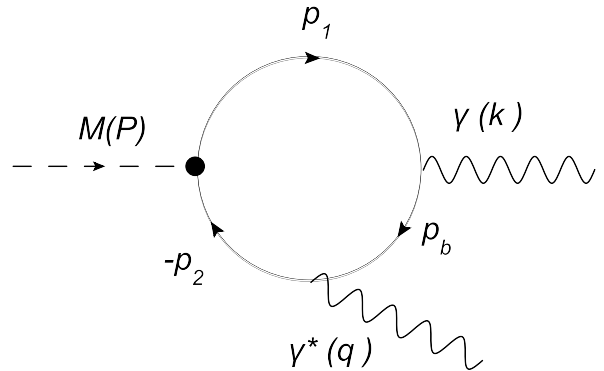

(b)

FIG. 1: Feynman diagrams for the $M \rightarrow \gamma \gamma^{*}$ process where $P$ in the parentheses denotes the momentum of the meson. (b) is related to (a) by the exchange of two photons.

the momentum $P=p_{1}+p_{2}$, where $p_{1}$ and $p_{2}$ are the momenta of the off-shell quark and antiquark, respectively, with masses $m$. These momenta can be expressed in terms of the internal variables $\left(x_{i}, p_{\perp}\right)$,

$$
p_{1,2}^{+}=x_{1,2} P^{+}, \quad p_{1,2 \perp}=x_{1,2} P_{\perp} \pm p_{\perp}
$$

with $x_{1}+x_{2}=1$. Note that we use $P=\left(P^{+}, P^{-}, P_{\perp}\right)$, where $P^{ \pm}=P^{0} \pm P^{3}$, so that $P^{2}=P^{+} P^{-}-P_{\perp}^{2}$. In the covariant light-front approach, total four momentum is conserved 
at each vertex where quarks and antiquarks are off shell. However, it is useful to define some internal quantities analogous to those defined for on-shell quarks:

$$
e=\sqrt{m^{2}+p_{\perp}^{2}+p_{z}^{2}}, \quad p_{z}=\frac{\left(x_{2}-x_{1}\right) M_{0}}{2}, \quad M_{0}^{2}=(2 e)^{2}=\frac{p_{\perp}^{2}+m^{2}}{x_{1} x_{2}} .
$$

where $m$ is the mass of heavy quark $c$ or $b$. Here, $M_{0}^{2}$ can be interpreted as the kinetic invariant mass squared of the incoming $q \bar{q}$ system and $e_{i}$ the energy of the quark $i$.

We need Feynman rules for the meson-quark-antiquark vertices to calculate the amplitudes shown in Fig. 1. These Feynman rules for vertices of scalar $(S)$ and tensor $(T)$ mesons are

$$
i \Gamma_{M}= \begin{cases}-i H_{S}, & \text { for }{ }^{3} P_{0}, \\ \frac{i}{2} H_{T}\left[\gamma_{\mu}-\frac{1}{W_{V}}\left(p_{1}-p_{2}\right)_{\mu}\right]\left(p_{1}-p_{2}\right)_{\nu}, & \text { for }{ }^{3} P_{2} .\end{cases}
$$

It has been shown in Ref. [35] that one can pass to the light-front approach by integrating out the $p^{-}$component of the internal momentum in covariant Feynman momentum loop integrals. The specific form of the covariant vertex functions for on-shell quarks can be determined through a comparison with the conventional vertex functions as shown in Appendix A of Ref. [33]. They are obtained as

$$
\begin{aligned}
& H_{M} \rightarrow H_{M}\left(\hat{p}_{1}^{2}, \hat{p}_{2}^{2}\right) \equiv h_{M}, \\
& W_{V} \rightarrow W_{V}\left(\hat{p}_{1}^{2}, \hat{p}_{2}^{2}\right) \equiv w_{V},
\end{aligned}
$$

where $\hat{p}_{1}=P-\hat{p}_{2}$ and $\hat{p}_{2}^{2}=m^{2}$. The form of the function $h_{M}$ contains two parts: one is the momentum distribution amplitude $\phi\left(x_{i}, p_{\perp}\right)$ which is the central ingredient in light-front QCD, while the other is a spin wave function which constructs a state of definite spin $\left(S, S_{z}\right)$ out of light-front helicity eigenstates $\left(\lambda_{1}, \lambda_{2}\right)$. The spin wave function is constructed by using the Melosh transformation [26] and its spin structure is shown in Eq. (2.3). The explicit forms of $h_{M}$ and $w_{V}$ are given by

$$
\begin{aligned}
& h_{S}=\left(M^{2}-M_{0}^{2}\right) \sqrt{\frac{x_{1} x_{2}}{N_{c}}} \frac{1}{\sqrt{2} M_{0}} \frac{M_{0}}{2 \sqrt{3}} \phi, \\
& h_{T}=\left(M^{2}-M_{0}^{2}\right) \sqrt{\frac{x_{1} x_{2}}{N_{c}}} \frac{1}{\sqrt{2} M_{0}} \phi, \\
& w_{V}=M_{0}+2 m .
\end{aligned}
$$

The momentum distribution amplitude $\phi\left(x_{i}, p_{\perp}\right)$ is the generalization of the distribution amplitude $\phi\left(x_{i}\right)$ of the pQCD method and can be chosen to be normalizable, i.e., it satisfies

$$
\int \frac{d x d^{2} p_{\perp}}{2(2 \pi)^{3}}\left|\phi\left(x, p_{\perp}\right)\right|^{2}=1
$$


In principle, $\phi\left(x, p_{\perp}\right)$ is obtained by solving the light-front QCD bound state equation $H_{L F}|\Psi\rangle=M|\Psi\rangle$ which is the familiar Schrödinger equation in ordinary quantum mechanics and $H_{L F}$ is the light-front Hamiltonian. However, at the present time, methods concerning how one can solve bound states equations are still unknown. We are satisfied with utilizing some phenomenological momentum distribution amplitudes which have been constructed phenomenologically in describing hadrons. One widely used form is the Gaussian type which we will employ in the application of the covariant light-front approach.

\section{B. Two-photon decay widths of $p$-wave heavy quarkonium}

Quarkonia are eigenstates of the charge conjugation operator $C$ with eigenvalues $C=$ $(-1)^{L+S}$, and the charge conservation requires charge conjugation $C=+1$ state coupling to two real photons. Thus, only ${ }^{3} P_{0}$ and ${ }^{3} P_{2}$ levels of the $p$-wave quarkonium states can transform into two real photons. In the process, $M \rightarrow \gamma \gamma$, the final two photons are both on shell. For the purpose of illustration, it is useful to consider a more general process: $M \rightarrow \gamma \gamma^{*}$ with one photon off shell. We introduce some transition form factors $f_{i}\left(q^{2}\right)$ arising from the $M \gamma \gamma^{*}$ vertex. The $M \rightarrow \gamma \gamma$ process is related to the form factors at $q^{2}=0$, i.e., $f_{i}(0)$. It is worth mentioning that, however, the state $M$ in the process $M \rightarrow \gamma \gamma^{*}$ is not only the ${ }^{3} P_{0}$ and ${ }^{3} P_{2}$ levels. [10]:

The matrix elements of the decay $S \rightarrow \gamma \gamma^{*}$ and $T \rightarrow \gamma \gamma^{*}$ have the following structures

$$
\begin{aligned}
\mathcal{A}_{\mu}^{S} & \equiv\left\langle\gamma(k, \epsilon)\left|\bar{q} \gamma_{\mu} q\right| S(P)\right\rangle=f_{0}\left(q^{2}\right)\left[\epsilon_{\mu}(P \cdot k)-k_{\mu}(P \cdot \epsilon)\right] \\
\mathcal{A}_{\mu}^{T} & \equiv\left\langle\gamma(k, \epsilon)\left|\bar{q} \gamma_{\mu} q\right| T(P, \varepsilon)\right\rangle \\
& =f_{1}\left(q^{2}\right)\left[\epsilon_{\mu}(q \cdot \varepsilon \cdot k)-k_{\mu}(q \cdot \varepsilon \cdot \epsilon)+\varepsilon_{\mu \nu} \epsilon^{\nu}(q \cdot k)-\varepsilon_{\mu \nu} k^{\nu}(\epsilon \cdot q)\right] \\
& +f_{2}\left(q^{2}\right)\left[\epsilon_{\mu}(q \cdot k)-k_{\mu}(\epsilon \cdot q)\right] \frac{q \cdot \varepsilon \cdot k}{q \cdot k}
\end{aligned}
$$

where $\epsilon$ is the polarization vector of the on-shell photon, $q=P-k$ is the momentum transfer, and $\varepsilon$ is the polarization of the tensor meson which satisfies the following relations:

$$
\begin{aligned}
& P^{\mu} \varepsilon_{\mu \nu}(\lambda)=P^{\nu} \varepsilon_{\mu \nu}(\lambda)=0, \quad \varepsilon_{\mu \nu}(\lambda)=\varepsilon_{\nu \mu}(\lambda), \quad \varepsilon_{\mu \mu}(\lambda)=0, \quad \varepsilon^{\mu \nu}(\lambda) \varepsilon_{\mu \nu}^{*}\left(\lambda^{\prime}\right)=\delta_{\lambda \lambda^{\prime}}, \\
& \sum_{\lambda} \varepsilon_{\mu \nu}(\lambda) \varepsilon_{\alpha \beta}^{*}(\lambda)=\frac{1}{2} M_{\mu \alpha} M_{\nu \beta}+\frac{1}{2} M_{\mu \beta} M_{\nu \alpha}-\frac{1}{3} M_{\mu \nu} M_{\alpha \beta},
\end{aligned}
$$


with $\lambda= \pm 2, \pm 1,0$ representing the tensor meson helicities and $M_{\mu \nu}=g_{\mu \nu}-P_{\mu} P_{\nu} / M^{2}$. There is no explicit representation of the meson polarization tensor $\varepsilon_{\mu \nu}(\lambda)$. The transition amplitude for the processes of $S \rightarrow \gamma \gamma^{*}$ and $T \rightarrow \gamma \gamma^{*}$ can be derived from the common Feynman rules and the vertices for the meson-quark-antiquark coupling given in Eq. (2.3). In the covariant light-front approach, the meson is on shell while the constituent quarks are off shell. To the lowest order approximation, $S(T) \rightarrow \gamma \gamma^{*}$ is a one-loop diagram and is depicted in Fig. 1. The amplitude is given as a momentum integral

$$
\begin{aligned}
& \mathcal{A}_{\mu}^{S}=i e_{q}^{2} e^{2} N_{c} \int \frac{d^{4} p_{1}}{(2 \pi)^{4}}\left\{\frac{H_{S}}{N_{1} N_{2} N_{a}} \operatorname{Tr}\left[\left(-\not p_{2}+m\right) \notin\left(\not \not_{a}+m\right) \gamma_{\mu}\left(\not p_{1}+m\right)\right]\right. \\
&\left.+\frac{H_{S}}{N_{1} N_{2} N_{b}} \operatorname{Tr}\left[\left(-\not p_{2}+m\right) \gamma_{\mu}\left(\not \not b_{b}+m\right) \notin\left(\not p_{1}+m\right)\right]\right\}, \\
& \mathcal{A}_{\mu}^{T}=i e_{q}^{2} e^{2} N_{c} \int \frac{d^{4} p_{1}}{(2 \pi)^{4}}\left\{\frac{H_{T}}{N_{1} N_{2} N_{a}} \operatorname{Tr}\left[t\left(-\not p_{2}+m\right) \notin\left(\not p_{a}+m\right) \gamma_{\mu}\left(\not p_{1}+m\right)\right]\right. \\
&\left.+\frac{H_{T}}{N_{1} N_{2} N_{b}} \operatorname{Tr}\left[t\left(-\not p_{2}+m\right) \gamma_{\mu}\left(\not p_{b}+m\right) \notin\left(\not p_{1}+m\right)\right]\right\},
\end{aligned}
$$

where

$$
\begin{aligned}
& p_{a}=p_{1}-q, \quad p_{b}=q-p_{2}, \\
& N_{1}=p_{1}^{2}-m^{2}+i \epsilon, \quad N_{2}=p_{2}^{2}-m^{2}+i \epsilon, \\
& N_{a}=p_{a}^{2}-m^{2}+i \epsilon, \quad N_{b}=p_{b}^{2}-m^{2}+i \epsilon, \\
& t=\varepsilon_{\alpha \beta}\left[\gamma^{\alpha}-\frac{1}{W_{V}}\left(p_{1}-p_{2}\right)^{\alpha}\right] \frac{\left(p_{1}-p_{2}\right)^{\beta}}{2},
\end{aligned}
$$

and $e_{q}$ is the electric charge of the quark: $e_{q}=2 / 3$ for the $c$ quark and $e_{q}=-1 / 3$ for the $b$ quark. The first and second terms in Eq. (2.10) come from diagrams Figs. 1 (a) and 1 (b), respectively. In the calculation, it is convenient to choose the purely transverse frame $q^{+}=0$, i.e., $q^{2}=-q_{\perp}^{2} \leq 0$. The advantage of this choice is that there is no so-called $Z$ - diagram contributions. The sacrifice associated with this approach is that only the form factor at spacelike regions can be calculated directly. The values at the timelike momentum transfer $q^{2}>0$ regions are obtained through analytic continuation. In this study, the continuation is not necessary because we only need the form factors at $q^{2}=0$ for the $S(T) \rightarrow \gamma \gamma$ process.

First, we discuss the calculation of Fig. 1 (a). The factors $N_{1}, N_{2}$, and $N_{a}$ produce three singularities in the $p_{1}^{-}$complex plane: one lies in the upper plane and the other two are in the lower plane. By closing the contour in the upper $p_{1}^{-}$complex plane, the momentum integral can be easily calculated since there is only one singularity in the plane. This corresponds 
to putting the antiquark on the mass shell. Given this restriction, the momentum $p_{2} \rightarrow \hat{p}_{2}$ with $\hat{p}_{2}^{2}=m^{2}$, and $\hat{p}_{1}=P-\hat{p}_{2}$. The on-shell restriction and the requirement of covariance lead to the following replacements:

$$
\begin{aligned}
N_{1} & \rightarrow \hat{N}_{1}=x_{1}\left(M^{2}-M_{0}^{2}\right), \\
N_{2} & \rightarrow \hat{N}_{2}=\hat{N}_{1}+\left(1-2 x_{1}\right) M^{2}=x_{2} M^{2}-x_{1} M_{0}^{2}, \\
N_{a} & \rightarrow \hat{N}_{a}=x_{2} q^{2}-x_{1} M_{0}^{2}+2 p_{\perp} \cdot q_{\perp}, \\
\int \frac{d^{4} p_{1}}{\left(2 \pi^{4}\right)} \frac{H_{S, T}}{N_{1} N_{2} N_{a}} & \rightarrow-i \pi \int \frac{d x_{2} d^{2} p_{\perp}}{\left(2 \pi^{4}\right)} \frac{h_{S, T}}{x_{2} \hat{N}_{1} \hat{N}_{a}} .
\end{aligned}
$$

For Fig. 1 (b), the contour is closed in the lower $p_{1}^{-}$complex plane. It corresponds to putting the quark on the mass shell and the momentum $p_{1} \rightarrow \hat{p}_{1}$ with $\hat{p}_{1}^{2}=m^{2}$. For this scenario, we need to do the following replacements:

$$
\begin{aligned}
N_{1} & \rightarrow \hat{N}_{1}=x_{1} M^{2}-x_{2} M_{0}^{2} x_{1}\left(M^{2}-M_{0}^{2}\right), \\
N_{2} & \rightarrow \hat{N}_{2}=x_{2}\left(M^{2}-M_{0}^{2}\right) \\
N_{b} & \rightarrow \hat{N}_{a}=x_{1} q^{2}-x_{2} M_{0}^{2}-2 p_{\perp} \cdot q_{\perp} \\
\int \frac{d^{4} p_{1}}{\left(2 \pi^{4}\right)} \frac{H_{S, T}}{N_{1} N_{2} N_{b}} & \rightarrow-i \pi \int \frac{d x_{2} d^{2} p_{\perp}}{\left(2 \pi^{4}\right)} \frac{h_{S, T}}{x_{1} \hat{N}_{2} \hat{N}_{b}} .
\end{aligned}
$$

After the above treatments, the transition amplitudes of $S \rightarrow \gamma \gamma^{*}$ and $T \rightarrow \gamma \gamma^{*}$ for Fig. 1 (a), for example, are obtained as

$$
\begin{aligned}
\mathcal{A}_{\mu}^{S(a)}= & N_{c} e_{q}^{2} e^{2} \int \frac{d x_{2} d^{2} p_{\perp}}{4 \pi^{3}} \frac{h_{S}}{x_{1} x_{2}\left(M^{2}-M_{0}^{2}\right)} \frac{M_{0}}{-x_{2} q^{2}+x_{1} M_{0}^{2}-2 p_{\perp} \cdot q_{\perp}} \frac{M^{2}}{2 \sqrt{3}} \\
\times & \left\{\epsilon_{\mu}\left(m^{2}-P \cdot q-p_{1}^{2}+2 p_{1} \cdot q\right)+2 p_{1 \mu}\left(2 \epsilon \cdot p_{1}-\epsilon \cdot P-\epsilon \cdot q\right)+q_{\mu}\left(\epsilon \cdot P-2 \epsilon \cdot p_{1}\right)\right. \\
& \left.+P_{\mu} \epsilon \cdot q\right\} \\
\mathcal{A}_{\mu}^{T(a)}= & N_{c} e_{q}^{2} e^{2} \int \frac{d x_{2} d^{2} p_{\perp}}{4 \pi^{3}} \frac{h_{T}}{x_{1} x_{2}\left(M^{2}-M_{0}^{2}\right)} \frac{1}{-x_{2} q^{2}+x_{1} M_{0}^{2}-2 p_{\perp} \cdot q_{\perp}} \\
\times & \left\{\epsilon_{\mu}\left[\left(m^{2}+P \cdot p_{1}-p_{1}^{2}\right) p_{1} \cdot \varepsilon \cdot q+\left(m^{2}-P \cdot q+2 p_{1} \cdot q-p_{1}^{2}\right)\left(1-\frac{2 m}{w_{V}}\right) p_{1} \cdot \varepsilon \cdot p_{1}\right]\right. \\
& +P_{\mu}\left[\left(m^{2}+p_{1} \cdot q-p_{1}^{2}\right) \epsilon \cdot \varepsilon \cdot p_{1}-\epsilon \cdot p_{1} p_{1} \cdot \varepsilon \cdot q+\epsilon \cdot q\left(1-\frac{2 m}{w_{V}}\right) p_{1} \cdot \varepsilon \cdot p_{1}\right] \\
& +q_{\mu}\left[\left(p_{1}^{2}-m^{2}-P \cdot p_{1}\right) \epsilon \cdot \varepsilon \cdot p_{1}+\left(\epsilon \cdot P-2 \epsilon \cdot p_{1}\right)\left(1-\frac{2 m}{w_{V}}\right) p_{1} \cdot \varepsilon \cdot p_{1}\right] \\
& +p_{1 \mu}\left[\left(m^{2}-P \cdot q+2 P \cdot p_{1}-p_{1}^{2}\right) \epsilon \cdot \varepsilon \cdot p_{1}+\epsilon \cdot P p_{1} \cdot \varepsilon \cdot q\right. \\
& \left.+\left(4 \epsilon \cdot p_{1}-2 \epsilon \cdot q-2 \epsilon \cdot P\right)\left(1-\frac{2 m}{w_{V}}\right) p_{1} \cdot \varepsilon \cdot p_{1}\right] \\
& +\varepsilon_{\mu \nu} p_{1}^{\nu}\left[m^{2}\left(\epsilon \cdot p_{1}-\epsilon \cdot P-\epsilon \cdot q\right)+\left(\epsilon \cdot p_{1} P \cdot q-\epsilon \cdot P p_{1} \cdot q-\epsilon \cdot q P \cdot p_{1}\right)\right.
\end{aligned}
$$




$$
\left.\left.+p_{1}^{2}\left(\epsilon \cdot q+\epsilon \cdot P-\epsilon \cdot p_{1}\right)\right]\right\}
$$

The integration of $p_{1 \mu}, p_{1 \mu} p_{1 \nu}, p_{1 \mu} p_{1 \nu} p_{1 \alpha}$, and $p_{1 \mu} p_{1 \nu} p_{1 \alpha} p_{1 \beta}$ in Eqs. (2.15) and (2.16) can be expressed in terms of three external vectors: $\widetilde{P}, q$, and $\omega$, as in the Appendix. The transition amplitude of $S(T) \rightarrow \gamma \gamma^{*}$ for Fig. 1 (b) can be obtained through a similar process. If we choose the frame where the meson is at rest and the photons travel in the $\pm z$ directions, then the two-photon decay amplitude of $S \rightarrow \gamma \gamma$ is obtained as

$$
\begin{aligned}
& \mathcal{M}^{S}(S \rightarrow \gamma \gamma)=\left.\left(\mathcal{A}_{\mu}^{S(a)}+\mathcal{A}_{\mu}^{S(b)}\right)\right|_{q^{2}=0} \cdot \epsilon^{\prime} \\
= & \left.e_{q}^{2} e^{2} \sqrt{\frac{N_{c}}{2}} \epsilon \cdot \epsilon^{\prime} \int \frac{d x_{2} d^{2} p_{\perp}}{8 \pi^{3}} \frac{m \phi}{\sqrt{m^{2}+p_{\perp}^{2}}}\left(\frac{2 x_{1} M_{0}^{2}-M^{2}-4 p_{\perp}^{2}}{2 \sqrt{3} x_{1} M_{0}}+\frac{2 x_{2} M_{0}^{2}-M^{2}-4 p_{\perp}^{2}}{2 \sqrt{3} x_{2} M_{0}}\right) .17\right)
\end{aligned}
$$

where $\epsilon^{\prime}$ is the polarization of another photon. The decay rate of the process $S \rightarrow \gamma \gamma$ is [36]

$$
\Gamma(S \rightarrow \gamma \gamma)=\frac{s}{8 \pi M_{S}^{2}}|\vec{k}| \sum_{\text {pol }}\left|\mathcal{M}^{S}\right|^{2}
$$

where $s=1 / 2$ for two identical photons and the photon momentum $|\vec{k}|=M / 2$. Regarding the case of $T \rightarrow \gamma \gamma$, in the practical calculations below, it is convenient to represent $\varepsilon_{\mu \nu}$ in the following form [37]:

$$
\begin{aligned}
\varepsilon_{\mu \nu}(\lambda)= & \frac{\sqrt{6}}{12}(2-|\lambda|)(1-|\lambda|)\left[3 n_{3}^{\mu} n_{3}^{\nu}+\left(g_{\mu \nu}-\frac{P_{\mu} P_{\nu}}{M^{2}}\right)\right] \\
& +\frac{1}{4}(1-|\lambda|)\left[\lambda\left(n_{1}^{\mu} n_{1}^{\nu}-n_{2}^{\mu} n_{2}^{\nu}\right)+i|\lambda|\left(n_{1}^{\mu} n_{2}^{\nu}+n_{2}^{\mu} n_{1}^{\nu}\right)\right] \\
& +\frac{1}{2}(2-|\lambda|)\left[\lambda\left(n_{1}^{\mu} n_{3}^{\nu}+n_{3}^{\mu} n_{1}^{\nu}\right)+i|\lambda|\left(n_{2}^{\mu} n_{3}^{\nu}+n_{2}^{\mu} n_{1}^{\nu}\right)\right],
\end{aligned}
$$

where $n_{i}^{\mu}(i=1,2,3)$ is the basis vector satisfying $n_{i}^{\mu} n_{j}^{\nu} g_{\mu \nu}=g_{i j}$. In the center-of-mass frame, we may have $P^{\mu}=(E, 0,0,|\mathbf{P}|)$ and choose the basis with collinear $n_{3}^{\mu}$ and $P^{\mu}$ vectors as the simplest one

$$
n_{1}^{\mu}=(0,1,0,0), \quad n_{2}^{\mu}=(0,0,1,0), \quad n_{3}^{\mu}=\frac{1}{M}(|\mathbf{P}|, 0,0, E)
$$

For the frame in which the meson is at rest and the photons travel in the $\pm z$ directions, the decay amplitudes of $T \rightarrow \gamma \gamma$ are obtained for $\lambda=0$ and \pm 2

$$
\begin{aligned}
\mathcal{M}_{\lambda=0}^{T}(T \rightarrow \gamma \gamma) & =\left.\left(\mathcal{A}_{\mu}^{T(a)}+\mathcal{A}_{\mu}^{T(b)}\right)\right|_{q^{2}=0, \lambda=0} \cdot \epsilon^{\prime} \\
& =e_{q}^{2} e^{2} \sqrt{\frac{N_{c}}{2}} \sqrt{\frac{2}{3}} \epsilon \cdot \epsilon^{\prime} \int \frac{d x_{2} d^{2} p_{\perp}}{16 \pi^{3}} \frac{\phi}{\sqrt{m^{2}+p_{\perp}^{2}}}\left(\frac{t_{0 a}}{x_{1} M_{0}^{2}}+\frac{t_{0 b}}{x_{2} M_{0}^{2}}\right)
\end{aligned}
$$




$$
\begin{aligned}
\mathcal{M}_{\lambda= \pm 2}^{T}(T \rightarrow \gamma \gamma) & =\left.\left(\mathcal{A}_{\mu}^{T(a)}+\mathcal{A}_{\mu}^{T(b)}\right)\right|_{q^{2}=0, \lambda= \pm 2} \cdot \epsilon^{\prime} \\
& =e_{q}^{2} e^{2} \sqrt{\frac{N_{c}}{2}} \int \frac{d x_{2} d^{2} p_{\perp}}{8 \pi^{3}} \frac{\phi}{\sqrt{m^{2}+p_{\perp}^{2}}}\left(\frac{t_{2 a}}{x_{1} M_{0}^{2}}+\frac{t_{2 b}}{x_{2} M_{0}^{2}}\right)
\end{aligned}
$$

where

$$
\begin{aligned}
t_{0 a} & =x_{1}\left(m^{2}-x_{2} M^{2}\right)\left(x_{1} M_{0}^{2}-x_{2} M^{2}\right)+p_{\perp}^{2}\left[-3 m^{2}+\left(1-4 x_{1}+2 x_{1}^{2}\right) M^{2}\right]-p_{\perp}^{4} \\
& +\frac{m}{w_{V}}\left\{x_{1}\left(2 m^{2}-M^{2}\right)\left(x_{2} M^{2}-x_{1} M_{0}^{2}\right)+p_{\perp}^{2}\left[2 m^{2}-4 x_{1}^{2} M_{0}^{2}+\left(4 x_{1} x_{2}-1\right) M^{2}\right]-2 p_{\perp}^{4}\right\}, \\
t_{0 b} & =\left.t_{0 a}\right|_{x_{1} \leftrightarrow x_{2}}, \\
t_{ \pm 2 a} & =p_{\perp}^{2}\left(m^{2}+M_{0}^{2} x_{1}^{2}+2 \frac{m}{w_{V}} p_{\perp}^{2}\right)\left(i \epsilon^{\prime} \cdot n_{2} \epsilon \cdot n_{1}+i \epsilon \cdot n_{2} \epsilon^{\prime} \cdot n_{1} \pm \epsilon^{\prime} \cdot n_{1} \epsilon \cdot n_{1} \mp \epsilon \cdot n_{2} \epsilon^{\prime} \cdot n_{2}\right), \\
t_{ \pm 2 b} & =\left.t_{2 a}\right|_{x_{1} \leftrightarrow x_{2}} .
\end{aligned}
$$

The derivations of Eqs. (2.21) and (2.22) use the formulas

$$
\int d^{2} p_{\perp}\left[p_{\perp} \cdot q_{\perp},\left(p_{\perp} \cdot q_{\perp}\right)^{2},\left(p_{\perp} \cdot q_{\perp}\right)^{3},\left(p_{\perp} \cdot q_{\perp}\right)^{4}\right]=\int d^{2} p_{\perp}\left[0, \frac{p_{\perp}^{2} q_{\perp}^{2}}{2}, 0, \frac{3 p_{\perp}^{4} q_{\perp}^{4}}{8}\right] .
$$

The amplitudes of $T \rightarrow \gamma \gamma$ for $\lambda= \pm 1$ vanish because the combination of the helicities of two final photons can be \pm 2 or 0 , but never equal to \pm 1 . The decay rate of the process $T \rightarrow \gamma \gamma$ is

$$
\Gamma(T \rightarrow \gamma \gamma)=\frac{s}{8 \pi M_{T}^{2}} \frac{|\vec{k}|}{5} \sum_{\lambda=0, \pm 1, \pm 2} \sum_{\mathrm{pol}}\left|\mathcal{M}^{T}\right|^{2}
$$

The factor 5 in the denominator corresponds to $2 J+1$, where $J$ is the total angular momentum of the meson.

Finally, the two-gluon decay width of quarkonium can be easily obtained from the twophoton decay width, with a simple replacement in the photon decay width formula

$$
e_{q}^{4} \alpha^{2} \rightarrow \frac{2}{9} \alpha_{s}^{2}
$$

\section{NUMERICAL RESULTS AND DISCUSSIONS}

In this section, the two-photon and two-gluon decay widths of $p$-wave heavy quarkonium states are estimated. Prior to numerical calculations, the parameters $m_{c, b}$ and $\beta_{c \bar{c}, b \bar{b}}$, which appeared in the wave function, must be first determined. We consider the Hamiltonian of the $s$ - and $p$-wave heavy quarkonium states as

$$
\begin{aligned}
& H_{s}=2 \sqrt{m^{2}+\vec{\kappa}^{2}}+V_{\text {conf }}-\frac{4 \alpha_{s}}{3 r}+g_{0} s_{1} \cdot s_{2}, \\
& H_{p}=2 \sqrt{m^{2}+\vec{\kappa}^{2}}+V_{\text {conf }}-\frac{4 \alpha_{s}}{3 r}+g_{1} S \cdot L+g_{2} S_{12}+g_{3} s_{1} \cdot s_{2},
\end{aligned}
$$


where $V_{\text {conf }}=b r\left(b r^{2}\right)$ is the linear [harmonic oscillator $(\mathrm{HO})$ ] potential, $S_{12}=\left(3 s_{1} \cdot \hat{r} s_{2} \cdot \hat{r}-\right.$ $\left.s_{1} \cdot s_{2}\right)$ is the tensor force operator, and $g_{0,1,2,3}$ are the functions of the relevant interquark potentials (the details are shown in, for example, [38, 39]). In this way, not only is the spin-weighted average of the $s$-wave states $M\left(S_{J}\right) \equiv\left[M\left({ }^{1} S_{0}\right)+3 M\left({ }^{3} S_{1}\right)\right] / 4$ free of the spinspin contribution, but also the mass difference between the spin-single ground state $M\left({ }^{1} P_{1}\right)$ and the spin-weighted average of the $p$-wave triplet states $M\left({ }^{3} P_{J}\right) \equiv\left[M\left({ }^{3} P_{0}\right)+3 M\left({ }^{3} P_{1}\right)+\right.$ $\left.5 M\left({ }^{3} P_{2}\right)\right] / 9$ has a contribution which comes from the spin-spin interaction. ${ }^{1}$ Experimentally the latter hyperfine splitting is less than $1 \mathrm{Mev}$ [1] in the charmonium sector and can be ignored here. Notably, we can use the masses $M\left(S_{J}\right), M\left({ }^{3} P_{J}\right)$ and their variational principle for the Hamiltonian equations. (3.1) and (3.2) to determine parameters $m$ and $\beta$. In the process, the $1 S, 1 P$, and $2 P$ states harmonic wave functions

$$
\begin{aligned}
& \phi^{1 S}\left(x, p_{\perp}\right)=4\left(\frac{\pi}{\beta^{2}}\right)^{3 / 4} \sqrt{\frac{d p_{z}}{d x}} \exp \left[-\frac{|\vec{p}|^{2}}{2 \beta^{2}}\right] \\
& \phi_{m}^{1 P}\left(x, p_{\perp}\right)=4\left(\frac{\pi}{\beta^{2}}\right)^{3 / 4} \sqrt{\frac{d p_{z}}{d x}} \sqrt{\frac{2}{\beta^{2}}} p_{m} \exp \left[-\frac{|\vec{p}|^{2}}{2 \beta^{2}}\right], \\
& \phi_{m}^{2 P}\left(x, p_{\perp}\right)=4\left(\frac{\pi}{\beta^{2}}\right)^{3 / 4} \sqrt{\frac{d p_{z}}{d x}} \sqrt{\frac{5}{\beta^{2}}} p_{m}\left(1-\frac{2|\vec{p}|^{2}}{5 \beta^{2}}\right) \exp \left[-\frac{|\vec{p}|^{2}}{2 \beta^{2}}\right],
\end{aligned}
$$

which satisfy the normalization Eq. (2.6) and their conjugate coordinate wave function

$$
\begin{aligned}
& \widetilde{\phi}^{1 S}(r)=\left(\frac{\beta^{2}}{\pi}\right)^{3 / 4} \exp \left[-\frac{\beta^{2} r^{2}}{2}\right], \\
& \widetilde{\phi}_{m}^{1 P}(r)=\sqrt{2}\left(\frac{\beta^{2}}{\pi}\right)^{3 / 4} \beta r_{m} \exp \left[-\frac{\beta^{2} r^{2}}{2}\right], \\
& \widetilde{\phi}_{m}^{2 P}(r)=\sqrt{5}\left(\frac{\beta^{2}}{\pi}\right)^{3 / 4} \beta r_{m}\left(1-\frac{2 \beta^{2} r^{2}}{5}\right) \exp \left[-\frac{\beta^{2} r^{2}}{2}\right],
\end{aligned}
$$

where $a_{m= \pm 1}=\mp\left(a_{x} \pm i a_{y}\right) / \sqrt{2}$ and $a_{m=0}=a_{z}$ are needed. In addition to the coefficient of confined potential $b$, the $c \bar{c}$ and $b \bar{b}$ sectors each have four parameters, $m_{q}, \alpha_{s}, \beta^{1 S}$, and $\beta^{1 P}$, for the $1 S$ and $1 P$ quarkonium states. Regarding the constraints, in addition to the four masses $M_{c \bar{c}, b \bar{b}}\left(1 S_{J}\right)$ and $M_{c \bar{c}, b \bar{b}}\left(1^{3} P_{J}\right)$, the four equations (3.3), (3.6) and (3.4), (3.7) are used as the trial functions of the variational principle for $1 S$ and $1 P$ states, respectively. Therefore, $b$ is the only free parameter in our fitting. We employ the data $\Gamma\left(\chi_{c 0} \rightarrow \gamma \gamma\right)=2.36 \pm 0.35 \mathrm{keV}$

\footnotetext{
${ }^{1}$ The calculations of the expectation values of the fourth and fifth terms for the Hamiltonian Eq. (3.2) can be referred to in the appendix of Ref. [40].
} 
TABLE I: The relevant parameters $b, m_{q}, \alpha_{s}$, and $\beta$ of the $p$-wave heavy quarkonium states.

\begin{tabular}{c|cccccc}
\hline \hline Potential & $b$ & & $m_{q}(\mathrm{GeV})$ & $\alpha_{s}$ & $\beta^{1 P}(\mathrm{GeV})$ & $\beta^{2 P}(\mathrm{GeV})$ \\
\hline Linear & $0.176_{-0.008}^{+0.007} \mathrm{GeV}^{2}$ & $c \bar{c}$ & $1.42 \mp 0.02$ & $0.489_{+0.019}^{-0.018}$ & $0.510 \pm 0.002$ & \\
& & $b \bar{b}$ & $4.78 \mp 0.01$ & $0.399_{+0.007}^{-0.006}$ & $0.807 \pm 0.005$ & $0.568_{-0.024}^{+0.025}$ \\
\hline \multirow{2}{*}{$\mathrm{HO}$} & $0.0490_{-0.0024}^{+0.0022} \mathrm{GeV}^{3}$ & $c \bar{c}$ & $1.42 \mp 0.02$ & $0.358_{+0.018}^{-0.025}$ & $0.577_{-0.003}^{+0.001}$ & \\
& & $b \bar{b}$ & $4.87_{+0.00}^{-0.01}$ & $0.425_{+0.006}^{-0.005}$ & $0.847 \pm 0.05$ & $0.571_{-0.009}^{+0.007}$ \\
\hline \hline
\end{tabular}

[1], to fix $b$; then the above parameters can be determined and shown in Table 1 . For $2 P$ states, due to the insufficient data regarding $\chi_{c J}^{\prime}$, only the parameter $\beta_{b \bar{b}}^{2 P}$ is determined by the mass $M_{b \bar{b}}\left(2^{3} P_{J}\right)$ and also revealed in Table 1 . There are three items in Table 1 worth mentioning: First, the parameter $b=0.176_{-0.008}^{+0.007} \mathrm{GeV}^{2}$ in the linear potential is consistent with the string tension $b=0.18 \mathrm{GeV}^{2}$ which is well known from other quark model analyses [12, 41, 42]. Second, there are presently different conceptions about the value of $\alpha_{s}$ in the low-energy region. In lattice QCD [43] and the field correlator method [39], for example, ones found for the coupling constant in the static potential, parametrized as a linear plus Coulomb potential, the small values $\alpha_{s}$ are 0.21 and 0.16 , respectively. In phenomenological potentials the Coulomb constant is larger: $\alpha_{s}$ is 0.46 [44] and $0.43 \pm 0.02$ [45] which corresponded to the charmonium and bottomonium states. Here our values are consistent with the latter. Third, in the HO potential, the strong coupling constant $\alpha_{s}$ of the $c \bar{c}$ sector is smaller than that of the $b \bar{b}$ sector, which violates the concept of asymptotic freedom. The dependences of $\alpha_{s}(c \bar{c})$ and $\alpha_{s}(b \bar{b})$ on $b$ regarding the linear and HO potentials are shown in Figs. 2 and 3, respectively. Obviously, the $\mathrm{HO}$ potential is not suitable when $b \geq 0.0410 \mathrm{GeV}^{3}$. However, in the data fitting of $\Gamma\left(\chi_{c 0} \rightarrow \gamma \gamma\right)$, we obtained $b=0.0490_{-0.0024}^{+0.0022} \mathrm{GeV}^{3}$. Therefore, only the linear potential is employed in the following calculations.

Next, we use the parameters of the linear potential in Table I and Eqs. (2.18), (2.26), and (2.27) to calculate the two-photon and two-gluon decay widths of the $\chi_{c 0, c 2}, \chi_{b 0, b 2}$, and $\chi_{b 0, b 2}^{\prime}$ states. The numerical results, which compare with the experimental data and other theoretical evaluations, are shown in Tables 2 and 3. From these tables, except in the case of our two-photon decay width of $\chi_{c 0}$ as an input, all the $c \bar{c}$ decay widths of this work are consistent with those of previous experiments and the major theoretical methods. However, our results of $\chi_{b 0, b 2}$ and $\chi_{b 0, b 2}^{\prime}$ differ from other calculations. More specifically, our 


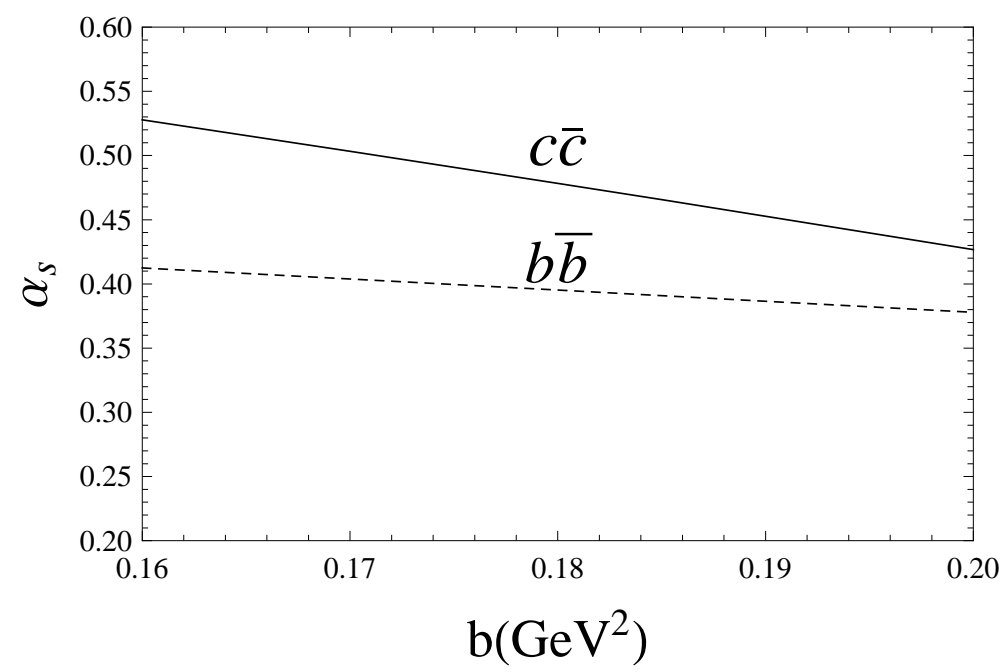

FIG. 2: Dependences of $\alpha_{s}(c \bar{c})$ (solid line) and $\alpha_{s}(b \bar{b})$ (dashed line) on $b$ in the linear potential

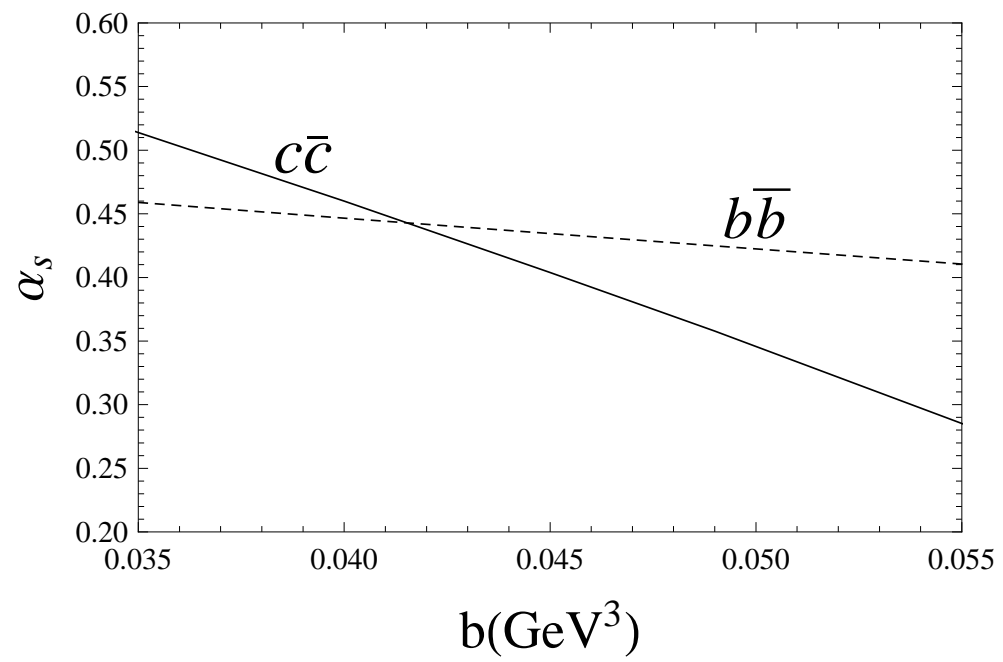

FIG. 3: Dependences of $\alpha_{s}(c \bar{c})$ (solid line) and $\alpha_{s}(b \bar{b})$ (dashed line) on $b$ in the HO potential.

two-photon decay widths of $\chi_{b 0, b 2}$ and $\chi_{b 0, b 2}^{\prime}$ are significantly smaller than those of other approaches. From Table 2, the ratio $\Gamma\left(\chi_{c 0} \rightarrow \gamma \gamma\right) / \Gamma\left(\chi_{b 0} \rightarrow \gamma \gamma\right)$ is about 300 for our work and about $60 \sim 80$ for other estimations. It is well known that the decay rate has two contributions: one is the kinematic phase space; the other is the dynamic decay amplitude square. No matter what approach, the phase space and factor $e_{q}^{4}$ of $\Gamma\left(\chi_{c 0} \rightarrow \gamma \gamma\right)$, in total, is about 50 times the one of $\Gamma\left(\chi_{b 0} \rightarrow \gamma \gamma\right)$. Regarding the comparison of decay amplitude $\mathcal{M}^{\mathcal{S}}$ in Eq. (2.17), we find that the contribution of the numerator in the parentheses is roughly the same for $\chi_{c 0}$ and $\chi_{b 0}$. On the other hand, the denominator in the parentheses, 
TABLE II: Two-photon widths of the $p$-wave heavy quarkonium states. $R$ is defined as $\Gamma_{2 \gamma}^{\chi_{c 2}} / \Gamma_{2 \gamma}^{\chi_{c 0}}$.

\begin{tabular}{|c|c|c|c|c|c|c|c|}
\hline & $\Gamma_{2 \gamma}^{\chi_{c 0}}(\mathrm{keV})$ & $\Gamma_{2 \gamma}^{\chi_{c 2}}(\mathrm{keV})$ & $\mathrm{R}$ & $\Gamma_{2 \gamma}^{\chi_{b 0}}(\mathrm{eV})$ & $\Gamma_{2 \gamma}^{\chi_{b 2}}(\mathrm{eV})$ & $\Gamma_{2 \gamma}^{\chi_{b 0}^{\prime}}(\mathrm{eV})$ & $\Gamma_{2 \gamma}^{\chi_{b 2}^{\prime}}(\mathrm{eV})$ \\
\hline PDG [1] & $2.36 \pm 0.35$ & $0.515 \pm 0.060$ & $0.218 \pm 0.058$ & & & & \\
\hline This work & $\underline{2.36 \pm 0.35}$ & $0.346_{-0.011}^{+0.009}$ & $0.147 \pm 0.026$ & $8.02_{-1.15}^{+1.12}$ & $3.99_{-0.13}^{+0.11}$ & $2.26_{-0.17}^{+0.16}$ & $1.72_{-0.33}^{+0.39}$ \\
\hline Münz [5] & $1.39 \pm 0.16$ & $0.44 \pm 0.14$ & $0.32_{-0.12}^{+0.16}$ & $24 \pm 3$ & $5.6 \pm 0.6$ & $26 \pm 2$ & $6.8 \pm 1.0$ \\
\hline Wang $[\underline{6}, \underline{7}]$ & 3.78 & 0.501 & 0.133 & 48.8 & 7.4 & 50.3 & 7.7 \\
\hline Ebert [10] & 2.9 & 0.50 & 0.17 & 38 & 8 & 29 & 6 \\
\hline Laverty $^{a}[\underline{8}]$ & $2.02(2.12)$ & $0.46(0.19)$ & $0.23(0.09)$ & $32.9(94.4)$ & $7.19(5.38)$ & $34.1(94.5)$ & $7.59(5.57)$ \\
\hline Schuler [13] & 2.5 & 0.28 & 0.11 & 43 & 7.4 & & \\
\hline Gupta $^{b}[9]$ & $6.38(8.13)$ & $0.57(1.14)$ & $0.09(0.14)$ & $80(85)$ & $8(12)$ & & \\
\hline Huang [18] & $3.72 \pm 1.11$ & $0.49 \pm 0.15$ & $0.13_{-0.06}^{+0.11}$ & & & & \\
\hline Bodwin [19] & $7.1 \pm 2.5$ & $0.81 \pm 0.29$ & $0.11 \pm 0.08$ & & & & \\
\hline Crater $^{c}[14]$ & $3.96(3.34)$ & $0.743(0.435)$ & $0.188(0.130)$ & & & & \\
\hline Barbieri [4] & 3.5 & 0.93 & 0.27 & & & & \\
\hline Godfrey [12] & 1.29 & 0.46 & 0.36 & & & & \\
\hline Lansberg [15] & 5.00 & 0.70 & 0.14 & & & & \\
\hline $\operatorname{Dudek}^{d}[17]$ & $2.41 \pm 0.58$ & & & & & & \\
\hline Lakhina [16] & 3.28 & & & & & & \\
\hline $\mathrm{LO}(\mathrm{NLO})^{e}[46]$ & & & $0.267(0.184)$ & & & & \\
\hline
\end{tabular}

${ }^{a}$ The values are obtained by the perturbative (nonperturbative) calculation.

${ }^{b}$ The values are obtained by the QCD potential (alternative treatment).

${ }^{c}$ The values are obtained by the two-body (naive) decay amplitude.

${ }^{d}$ Only the statistical error is shown.

${ }^{e}$ These values refer to a leading (next-to-leading) order calculation done at the renormalization scale $2 m_{c}$.

$M_{0}$, is approximately equal to the meson mass for the heavy quarkonium. Therefore, the dynamic decay amplitude square of $\chi_{c 0} \rightarrow \gamma \gamma$ is about 6 times that of $\chi_{b 0} \rightarrow \gamma \gamma$. Our ratio $\Gamma\left(\chi_{c 0} \rightarrow \gamma \gamma\right) / \Gamma\left(\chi_{b 0} \rightarrow \gamma \gamma\right) \simeq 300$ is obtained by combining the above two components. Regardless, more experimental measurements are required for these channels. Finally, the ratio $R \equiv \Gamma_{2 \gamma}^{\chi_{c 2}} / \Gamma_{2 \gamma}^{\chi_{c 0}}$ of experiment and various theoretical estimations are also listed in Table 2 for a comparison. 
TABLE III: Two-gluon decay widths of the $p$-wave heavy quarkonium states.

\begin{tabular}{|c|c|c|c|c|c|c|}
\hline & $\Gamma_{2 g}^{\chi_{c 0}}(\mathrm{MeV})$ & $\Gamma_{2 g}^{\chi_{c 2}}(\mathrm{MeV})$ & $\Gamma_{2 g}^{\chi_{b 0}}(\mathrm{keV})$ & $\Gamma_{2 g}^{\chi_{b 2}}(\mathrm{keV})$ & $\Gamma_{2 g}^{\chi_{b 0}^{\prime}}(\mathrm{keV})$ & $\Gamma_{2 g}^{\chi_{b 2}^{\prime}}(\mathrm{keV})$ \\
\hline $\mathrm{PDG}^{a}[1]$ & $10.4 \pm 0.7$ & $1.98 \pm 0.11$ & & & & \\
\hline This work & $11.9_{-0.9}^{+0.7}$ & $1.74_{+0.09}^{-0.08}$ & $431_{-49}^{+45}$ & $214_{+1}^{-0}$ & $122_{-6}^{+4}$ & $92.3_{-14.8}^{+17.7}$ \\
\hline Wang $[6,7]$ & 10.3 & 2.64 & 887 & 220 & 914 & 248 \\
\hline Laverty $^{b}$ [8] & $4.68(4.88)$ & $1.72(0.69)$ & $960(2740)$ & $330(250)$ & $990(2740)$ & $350(260)$ \\
\hline Gupta $^{c}[9]$ & $13.44(17.10)$ & $1.20(2.39)$ & $2150(2290)$ & $220(330)$ & & \\
\hline Bodwin [19] & $4.8 \pm 0.7$ & $\underline{1.98 \pm 0.18}$ & & & & \\
\hline Barbieri [4] & 2.4 & 0.64 & & & & \\
\hline Godfrey [12] & 6.25 & 0.774 & 672 & 123 & 672 & 137 \\
\hline Ebert [11] & & & 653 & 109 & 431 & 76 \\
\hline
\end{tabular}

\section{CONCLUSIONS}

This study has discussed two-photon and two-gluon decay widths of $p$-wave heavy quarkonium states within the covariant light-front quark model. This formalism, which preserves the Lorentz covariance in the light-front framework, was applied to annihilations of the scalar and tensor quarkonia. To obtain the numerical results, we used the harmonic wave functions and fixed the parameters appearing in them. The constraints were the spin-weighted average masses $M\left(S_{J},{ }^{3} P_{J}\right)$ and their variational principle regarding the Hamiltonian. We considered the linear and HO potentials in the Hamiltonian and found that, when the data $\Gamma\left(\chi_{c 0} \rightarrow \gamma \gamma\right)$ were fitted, the former resulted in a value of $b$ consistent with that of other quark models and the latter led to a violation of asymptotic freedom. Therefore, only the parameters corresponding to the linear potential were applied to estimate the relevant decay widths. The numerical results showed that, for the $c \bar{c}$ sector, all of the decay widths were in agreement with the experimental data and the major theoretical calculations. However, for the $b \bar{b}$ sector, discrepancies appeared in the decay widths of $\chi_{b 0, b 2}$ and $\chi_{b 0, b 2}^{\prime}$ from other estimations. 


\section{Acknowledgements}

This work is supported in part by the National Science Council of R.O.C. under Grant No NSC-99-2112-M-017-002-MY3.

\section{Appendix A: Formulas for the product of several $p_{1}$ 's}

In general, after the $p_{1}$ integration, $p_{1}$ can be expressed in terms of three external vectors, $\widetilde{P}, q$, and $\omega$, where $\widetilde{P}=P+k$. Furthermore, the inclusion of the zero- mode contribution cancels the $\omega$ dependence and in practice for $p_{1}$ in the trace under the integration, we have [32, 33]

$$
\begin{aligned}
\hat{p}_{1 \mu} \doteq & \widetilde{P}_{\mu} A_{1}^{(1)}+q_{\mu} A_{2}^{(1)} \\
\hat{p}_{1 \mu} \hat{p}_{1 \nu} \doteq & \left.g_{\mu \nu} A_{1}^{(2)}+\widetilde{P}_{\mu} \widetilde{P}_{\nu} A_{2}^{(2)}+\left(\widetilde{P}_{\mu} q_{\nu}+q_{\mu} \widetilde{P}_{\nu}\right) A_{3}^{(2)}+q_{\mu} q_{\nu} A_{4}^{(2)}+\frac{\widetilde{P}_{\mu} \omega_{\nu}+\omega_{\mu} \widetilde{P}_{\nu}}{\omega \widetilde{P}} B_{1}^{(2)} \mathrm{A} 2\right) \\
\hat{p}_{1 \mu} \hat{p}_{1 \nu} \hat{p}_{1 \alpha} \doteq & \left(g_{\mu \nu} \widetilde{P}_{\alpha}+g_{\mu \alpha} \widetilde{P}_{\nu}+g_{\nu \alpha} \widetilde{P}_{\mu}\right) A_{1}^{(3)}+\left(g_{\mu \nu} q_{\alpha}+g_{\mu \alpha} q_{\nu}+g_{\nu \alpha} q_{\mu}\right) A_{2}^{(3)}+\widetilde{P}_{\mu} \widetilde{P}_{\nu} \widetilde{P}_{\alpha} A_{3}^{(3)} \\
& +\left(\widetilde{P}_{\mu} \widetilde{P}_{\nu} q_{\alpha}+\widetilde{P}_{\mu} q_{\nu} \widetilde{P}_{\alpha}+q_{\mu} \widetilde{P}_{\nu} \widetilde{P}_{\alpha}\right) A_{4}^{(3)}+\left(q_{\mu} q_{\nu} \widetilde{P}_{\alpha}+q_{\mu} \widetilde{P}_{\nu} q_{\alpha}+\widetilde{P}_{\mu} q_{\nu} q_{\alpha}\right) A_{5}^{(3)} \\
& +q_{\mu} q_{\nu} q_{\alpha} A_{6}^{(3)}+\frac{1}{\omega \widetilde{P}}\left(\widetilde{P}_{\mu} \widetilde{P}_{\nu} \omega_{\alpha}+\widetilde{P}_{\mu} \omega_{\nu} \widetilde{P}_{\alpha}+\omega_{\mu} \widetilde{P}_{\nu} \widetilde{P}_{\alpha}\right) B_{1}^{(3)} \\
& +\frac{1}{\omega \widetilde{P}}\left[\left(\widetilde{P}_{\mu} q_{\nu}+q_{\mu} \widetilde{P}_{\nu}\right) \omega_{\alpha}+\left(\widetilde{P}_{\mu} q_{\alpha}+q_{\mu} \widetilde{P}_{\alpha}\right) \omega_{\nu}\left(\widetilde{P}_{\nu} q_{\alpha}+q_{\nu} \widetilde{P}_{\alpha}\right) \omega_{\mu}\right] B_{2}^{(3)} \\
\hat{p}_{1 \mu} \hat{p}_{1 \nu} \hat{p}_{1 \alpha} \hat{p}_{1 \beta} \doteq & \sum_{i=1}^{9} I_{i \mu \nu \alpha \beta} A_{i}^{(4)}+\sum_{j=1}^{4} J_{j \mu \nu \alpha \beta} B_{j}^{(4)}
\end{aligned}
$$

where the symbol $\doteq$ denotes that the equation is true only in the integration and

$$
\begin{aligned}
& I_{1 \mu \nu \alpha \beta}=g_{\mu \nu} g_{\alpha \beta}+g_{\mu \alpha} g_{\nu \beta}+g_{\mu \beta} g_{\nu \alpha}, \\
& I_{2 \mu \nu \alpha \beta}=g_{\mu \nu} \widetilde{P}_{\alpha} \widetilde{P}_{\beta}+g_{\mu \alpha} \widetilde{P}_{\nu} \widetilde{P}_{\beta}+g_{\mu \beta} \widetilde{P}_{\nu} \widetilde{P}_{\alpha}+g_{\alpha \beta} \widetilde{P}_{\mu} \widetilde{P}_{\nu}+g_{\nu \beta} \widetilde{P}_{\mu} \widetilde{P}_{\alpha}+g_{\nu \alpha} \widetilde{P}_{\mu} \widetilde{P}_{\beta}, \\
& I_{3 \mu \nu \alpha \beta}=g_{\mu \nu}\left(\widetilde{P}_{\alpha} q_{\beta}+\widetilde{P}_{\beta} q_{\alpha}\right)+\text { permutations, } \\
& I_{4 \mu \nu \alpha \beta}=g_{\mu \nu} q_{\alpha} q_{\beta}+g_{\mu \alpha} q_{\nu} q_{\beta}+g_{\mu \beta} q_{\nu} q_{\alpha}+g_{\alpha \beta} q_{\mu} q_{\nu}+g_{\nu \beta} q_{\mu} q_{\alpha}+g_{\nu \alpha} q_{\mu} q_{\beta}, \\
& I_{5 \mu \nu \alpha \beta}=\widetilde{P}_{\mu} \widetilde{P}_{\nu} \widetilde{P}_{\alpha} \widetilde{P}_{\beta}, \\
& I_{6 \mu \nu \alpha \beta}=\widetilde{P}_{\mu} \widetilde{P}_{\nu} \widetilde{P}_{\alpha} q_{\beta}+\widetilde{P}_{\mu} \widetilde{P}_{\nu} q_{\alpha} \widetilde{P}_{\beta}+\widetilde{P}_{\mu} q_{\nu} \widetilde{P}_{\alpha} \widetilde{P}_{\beta}+q_{\mu} \widetilde{P}_{\nu} \widetilde{P}_{\alpha} \widetilde{P}_{\beta}, \\
& I_{7 \mu \nu \alpha \beta}=\widetilde{P}_{\mu} \widetilde{P}_{\nu} q_{\alpha} q_{\beta}+\text { permutations, } \\
& I_{8 \mu \nu \alpha \beta}=\widetilde{P}_{\mu} q_{\nu} q_{\alpha} q_{\beta}+q_{\mu} \widetilde{P}_{\nu} q_{\alpha} q_{\beta}+q_{\mu} q_{\nu} \widetilde{P}_{\alpha} q_{\beta}+q_{\mu} q_{\nu} q_{\alpha} \widetilde{P}_{\beta}, \\
& I_{9 \mu \nu \alpha \beta}=q_{\mu} q_{\nu} q_{\alpha} q_{\beta}, \\
& J_{1 \mu \nu \alpha \beta}=\frac{1}{\omega \widetilde{P}}\left[g_{\mu \nu}\left(\widetilde{P}_{\alpha} \omega_{\beta}+\widetilde{P}_{\beta} \omega_{\alpha}\right)+\text { permutations }\right],
\end{aligned}
$$




$$
\begin{aligned}
& J_{2 \mu \nu \alpha \beta}=\frac{1}{\omega \widetilde{P}}\left(\widetilde{P}_{\mu} \widetilde{P}_{\nu} \widetilde{P}_{\alpha} \omega_{\beta}+\widetilde{P}_{\mu} \widetilde{P}_{\nu} \omega_{\alpha} \widetilde{P}_{\beta}+\widetilde{P}_{\mu} \omega_{\nu} \widetilde{P}_{\alpha} \widetilde{P}_{\beta}+\omega_{\mu} \widetilde{P}_{\nu} \widetilde{P}_{\alpha} \widetilde{P}_{\beta}\right), \\
& J_{3 \mu \nu \alpha \beta}=\frac{1}{\omega \widetilde{P}}\left[\left(\widetilde{P}_{\mu} \widetilde{P}_{\nu} q_{\alpha}+\widetilde{P}_{\mu} q_{\nu} \widetilde{P}_{\alpha}+q_{\mu} \widetilde{P}_{\nu} \widetilde{P}_{\alpha}\right) \omega_{\beta}+\text { permutations }\right] \\
& J_{4 \mu \nu \alpha \beta}=\frac{1}{\omega \widetilde{P}}\left[\left(\widetilde{P}_{\mu} q_{\nu} q_{\alpha}+q_{\mu} \widetilde{P}_{\nu} q_{\alpha}+q_{\mu} q_{\nu} \widetilde{P}_{\alpha}\right) \omega_{\beta}+\text { permutations }\right]
\end{aligned}
$$

with

$$
\begin{aligned}
& A_{1}^{(1)}=\frac{x_{1}}{2}, \quad A_{2}^{(1)}=A_{1}^{(1)}-\frac{p_{\perp} \cdot q_{\perp}}{q^{2}}, \quad A_{1}^{(2)}=-p_{\perp}^{2}-\frac{\left(p_{\perp} \cdot q_{\perp}\right)^{2}}{q^{2}}, \quad A_{2}^{(2)}=\left(A_{1}^{(1)}\right)^{2}, \\
& A_{3}^{(2)}=A_{1}^{(1)} A_{2}^{(1)}, \quad A_{4}^{(2)}=\left(A_{2}^{(1)}\right)^{2}-\frac{A_{1}^{(2)}}{q^{2}}, \quad B_{1}^{(2)}=A_{1}^{(1)} Z_{2}-A_{1}^{(2)}, \quad A_{1}^{(3)}=A_{1}^{(1)} A_{1}^{(2)}, \\
& A_{2}^{(3)}=A_{2}^{(1)} A_{1}^{(2)}, \quad A_{3}^{(3)}=A_{1}^{(1)} A_{2}^{(2)}, \quad A_{4}^{(3)}=A_{2}^{(1)} A_{2}^{(2)}, \quad A_{5}^{(3)}=A_{1}^{(1)} A_{4}^{(2)}, \\
& A_{6}^{(3)}=A_{2}^{(1)} A_{4}^{(2)}-\frac{2}{q^{2}} A_{2}^{(1)} A_{1}^{(2)}, \quad B_{1}^{(3)}=A_{1}^{(1)}\left(B_{1}^{(2)}-A_{1}^{(2)}\right), \quad B_{2}^{(3)}=A_{2}^{(1)} B_{1}^{(2)}+\frac{q \cdot \widetilde{P}}{q^{2}} A_{1}^{(3)}, \\
& A_{1}^{(4)}=\frac{\left(A_{1}^{(2)}\right)^{2}}{3}, \quad A_{2}^{(4)}=A_{1}^{(1)} A_{1}^{(3)}, \quad A_{3}^{(4)}=A_{1}^{(1)} A_{2}^{(3)}, \quad A_{4}^{(4)}=A_{2}^{(1)} A_{2}^{(3)}-\frac{A_{1}^{(4)}}{q^{2}}, \\
& A_{5}^{(4)}=A_{1}^{(1)} A_{3}^{(3)}, \quad A_{6}^{(4)}=A_{1}^{(1)} A_{4}^{(3)}, \quad A_{7}^{(4)}=A_{1}^{(1)} A_{5}^{(3)}, \quad A_{8}^{(4)}=A_{1}^{(1)} A_{6}^{(3)}, \\
& A_{9}^{(4)}=A_{1}^{(1)} A_{6}^{(3)}-\frac{3}{q^{2}} A_{4}^{(4)}, \quad B_{1}^{(4)}=A_{1}^{(1)} A_{1}^{(2)} Z_{2}-A_{1}^{(4)}, \quad B_{2}^{(4)}=A_{1}^{(1)} B_{1}^{(3)}-A_{2}^{(4)}, \\
& B_{3}^{(4)}=A_{1}^{(1)} B_{2}^{(3)}-A_{3}^{(4)}, \quad B_{4}^{(4)}=A_{1}^{(1)}\left(A_{4}^{(2)} Z_{2}+2 \frac{q \cdot \widetilde{P}}{q^{2}} A_{2}^{(1)} A_{1}^{(2)}\right)-A_{4}^{(4)}, \\
& Z_{2}=\hat{N}_{1}+\left(x_{2}-x_{1}\right) M^{2}+\left(q^{2}+q \cdot \widetilde{P}\right) \frac{p_{\perp} \cdot q_{\perp}}{q^{2}} .
\end{aligned}
$$

Equation (A6) is obtained by contracting the larger number of $\hat{p}_{1}$ 's with $\omega_{\beta}, q_{\beta}$, and $g_{\alpha \beta}$, and through a comparison with the complete expression of the fewer $\hat{p}_{1}$ 's.

[1] C. Amsler et al. (Particle Data Group), Phys. Lett. B 667, 1 (2008) and 2009 partial update for the 2010 edition.

[2] K. M. Ecklund et al., (CLEO Collaboration), Phys. Rev. D78, 091501 (2008) and references therein.

[3] T. Appelquist and H. D. Politzer, Phys. Rev. Lett. 34, 43 (1975).

[4] R. Barbieri, R. Gatto, and R. Kögerler, Phys. Lett. B60, 183 (1976).

[5] C. R. Münz, Nucl. Phys. A609, 364 (1996).

[6] G. L. Wang, Phys. Lett. B653, 206 (2007).

[7] G. L. Wang, Phys. Lett. B674, 172 (2009). 
[8] J. T. Laverty, S. F. Radford, and W. W. Repko, arXiv:0901.3917 [hep-ph].

[9] S. N. Gupta, J. M. Johnson, and W. W. Repko, Phys. Rev. D54, 2075 (1996).

[10] D. Ebert, R. N. Faustov, and V. O. Galkin, Mod. Phys. Lett. A18, 601 (2003).

[11] D. Ebert, R. N. Faustov, and V. O. Galkin, Phys. Rev. D67, 014027 (2003).

[12] S. Godfrey and N. Isgur, Phys. Rev. D32, 189 (1985).

[13] G. A. Schuler, F. A. Berends, and R. van Gulik, Nucl. Phys. B523, 423 (1998).

[14] H. W. Crater, C. Y. Wong, and P. VanAlstine, Phys. Rev. D74, 054028 (2006).

[15] J. P. Lansberg and T. N. Pham, Phys. Rev. D79, 094016 (2009).

[16] O. Lakhina and E. S. Swanson, Phys. Rev. D74, 014012 (2006).

[17] J. J. Dudek and R. G. Edwards, Phys. Rev. Lett. 97, 172001 (2006).

[18] H. W. Huang and K. T. Chao, Phys. Rev. D54, 6850 (1996); Erratum-ibid. D56, 1821 (1997).

[19] G. T. Bodwin, E. Braaten, and G. P. Lepage, Phys. Rev. D46, R1914 (1992).

[20] G. T. Bodwin, J. Lee, and D. K. Sinclair, Phys. Rev. D72, 014009 (2005).

[21] N. Brambilla, E. Mereghetti, and A. Vairo, JHEP 0608, 039 (2006).

[22] N. Brambilla, A. Vairo, and E. Mereghetti, Phys. Rev. D79, 074002 (2009).

[23] C. Quigg and J. L. Rosner, Phys. Rept. 56, 167-235 (1979).

[24] M. V. Terent'ev, Sov. J. Nucl. Phys. 24, 106 (1976); V. B. Berestetsky and M. V. Terent'ev, ibid 24, 547 (1976); 25, 347 (1977).

[25] P. L. Chung, F. Coester, and W. N. Polyzou, Phys. Lett. B205, 545 (1988).

[26] H. J. Melosh, Phys. Rev. D9, 1095 (1974).

[27] W. Jaus, Phys. Rev. D41, 3394 (1990).

[28] W. Jaus, Phys. Rev. D44, 2851 (1991).

[29] C. R. Ji, P. L. Chung, and S. R. Cotanch, Phys. Rev. D45, 4214 (1992)

[30] H. Y. Cheng, C. Y. Cheung, and C. W. Hwang, Phys. Rev. D55, 1559 (1997).

[31] H. Y. Cheng, C. Y. Cheung, C. W. Hwang, and W. M. Zhang, Phys. Rev. D57, 5598 (1998).

[32] W. Jaus, Phys. Rev. D60, 054026 (1999).

[33] H. Y. Cheng, C. K. Chua, and C. W. Hwang, Phys. Rev. D69, 074025 (2004).

[34] C. W. Hwang and Z. T. Wei, J. Phys. G34, 687 (2007).

[35] S. J. Chang and S. K. Ma, Phys. Rev. 180, 1506 (1969).

[36] H. M. Choi, arXiv:hep-ph/9911271.

[37] R. S. Pasechnik, A. Szczurek, and O. V. Teryaev, Phys. Rev. D81, 034024 (2010). 
[38] S. F. Radford and W. W. Repko, Phys. Rev. D75, 074031 (2007).

[39] A. M. Badalian, A. V. Nefediev, and Yu. A. Simonov, Phys. Rev. D78, 114020 (2008).

[40] R. N. Cahn and J. D. Jackson, Phys. Rev. D68, 037502 (2003).

[41] N. Isgur, D. Scora, B. Grinstein, and M. B. Wise, Phys. Rev. D39, 799 (1989).

[42] D. Scora and N. Isgur, Phys. Rev. D52, 2783 (1995).

[43] Y. Koma and M. Koma, Nucl. Phys. B769, 79 (2007).

[44] E. J. Eichten, K. Lane, and C. Quigg, Phys. Rev. Lett. 89, 162002 (2002).

[45] A. M. Badalian, A. I. Veselov, and B. L. G. Bakker, Phys. Rev. D70, 016007 (2004).

[46] N. Brambilla et al., arXiv:hep-ph/0412158. 